\title{
Wie man Ärger vermeidet
}

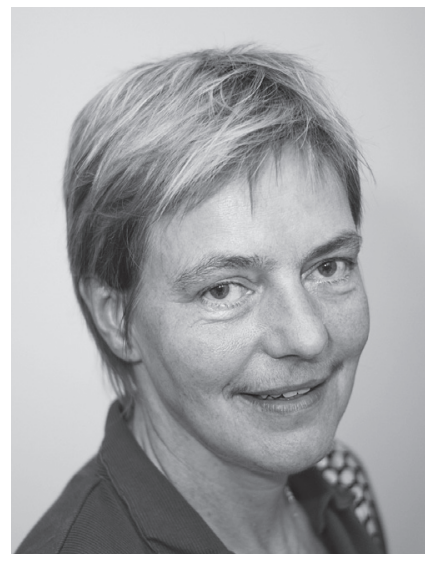

VON GERTRUD TACKE

Die Rechtsanwältin Gertrud Tacke ist seit 1993 im Deutschen Paritätischen Wohlfahrtsverband Gesamtverband e. V. in der Rechtsabteilung tätig. Ihr Schwerpunkt liegt im Arbeits- und Zivilrecht. Sie war Mitglied der Verhandlungsgruppe der Bundesarbeitsgemeinschaft Freie Wohlfahrtspflege, die die Gesamtverträge mit der GEMA und der VG Media aushandelte.

E-Mail

arbeitsrecht@paritaet.org

\author{
Viele soziale Organisationen und gemeinnützige \\ Unternehmen sehen sich mit Forderungen von \\ Verwertungsgesellschaften, beispielsweise der \\ GEMA und der VG Media, manchmal auch von \\ unbekannten Anbietern konfrontiert, die Geld \\ für das Weitersenden von Programmsignalen, für \\ das Kopieren von Musiknoten in Kitas oder das \\ Vorführen von Filmen eintreiben wollen. Für die \\ Wohlfahrtsverbände und ihre Mitgliedsorganisationen \\ gibt es dazu eine Reihe von Gesamtverträgen.
}

Die Nutzung von Musikwerken, Musiknoten und literarischen Texten ist in vielen sozialen Einrichtungen üblich, von Livekonzerten bis zu Filmvorführungen per DVD. In Heimen und Vereinsräumen stehen Fernseh- und Rundfunkgeräte und Abspielgeräte, über die Musik und Texte wiedergeben werden. Im Gegensatz zur privaten Nutzung ist in der Regel die öffentliche Wiedergabe von Werken geistigen Eigentums vergütungspflichtig.

Die Rechte der Urheber an ihren Werken sind nach dem Urheberrechtsgesetz geschützt. Das Urheberrecht versucht, einen Ausgleich zwischen den verschiedenen Akteuren in diesem Wirtschaftsbereich herzustellen. Dem Urheber eines Werkes steht das originäre Recht zur Verwertung, aber auch eine angemessene Beteiligung an der Auswertung zu.

Daneben stehen die Interessen der Verwertungsindustrie, die die schöpferischen Leistungen der Öffentlichkeit und damit dem Werknutzer erst zugänglich machen. Das Interesse der Werknutzer auf einen möglichst ungehinderten $\mathrm{Zu}$ gang zu den urheberrechtlichen Nutzungsmöglichkeiten zu einem akzeptablen Preis findet ebenfalls im Urheberrecht ihren Niederschlag.
Schließlich ist das Allgemeininteresse von Bedeutung. So sind auch soziale Aspekte, beispielsweise bei der Bemessung der Vergütungen zu berücksichtigen. Das Gesetz sieht in $\mathbb{} 52$ Urhebergesetz unter engen Bedingungen eine ausdrückliche Befreiung von der Vergütungs- und Erlaubnispflicht der öffentlichen Wiedergabe bei sozialen Veranstaltungen der Wohlfahrtspflege vor.

Dieser Rechtsbereich tut sich schwer, mit der sich rasant entwickelnden digitalen Welt mitzuhalten. Dies wird auch von den verschiedenen Interessenlagern beklagt. Die Rechtslage ist komplex, viele Rechtsfragen sind nicht abschließend geklärt. Nachfolgend soll versucht werden, ein wenig Licht in den Dschungel zu bringen.

\section{Wer GEMA, VG Media und Co. eigentlich sind}

In Deutschland gibt es derzeit zwölf verschiedene Verwertungsgesellschaften, welche treuhänderisch für eine große Anzahl von Urhebern oder Inhabern verwandter Schutzrechte unterschiedliche Rechte Dritter geltend machen. Sie stehen unter der staatlichen Aufsicht des Deutschen Marken- und Patentam- 


\section{Worauf zu achten ist}

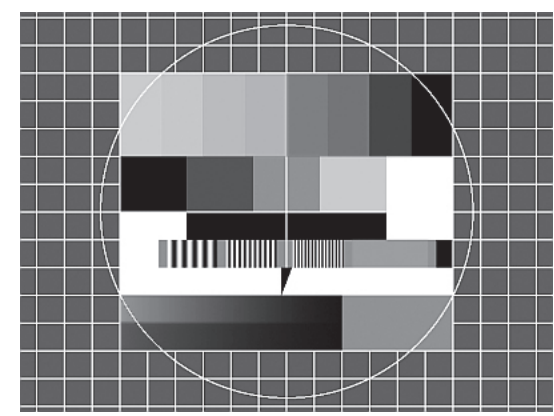

Soziale Veranstaltungen der Wohlfahrtspflege sind unter bestimmten Voraussetzungen von der Vergütungspflicht nach dem Gesetz über Urheberrecht und verwandte Schutzrechte befreit. Dazu müssen sämtliche folgende Bedingungen erfüllt sein:

- Es handelt sich um eine Veranstaltung der Jugendhilfe, Sozialhilfe, der Altenund Wohlfahrtspflege, der Gefangenenbetreuung sowie Schulveranstaltungen.
- Die Veranstaltung ist nach ihrer sozialen und erzieherischen Zweckbestimmung nur einem bestimmt abgrenzbaren Personenkreis zugänglich. Das ist der Fall, wenn der Zugang zu der Veranstaltung tatsächlich begrenzt ist und nicht beliebigen Dritten offen steht, beispielsweise Weihnachtsfeier, sonstige Feste oder Veranstaltungen, die sich ausschließlich an die Bewohner, Angehörige und Mitarbeitende richten, nicht aber beispielsweise der Tag der offenen Tür oder wenn die ganze Nachbarschaft eingeladen ist.

- Die Veranstaltung dient keinem Erwerbszweck des Veranstalters oder eines Dritten.

- Die Teilnehmenden müssen ohne Entgelt zugelassen sein.

- Der Künstler tritt ohne Entgelt auf.

Der Wortlaut des Gesetzes ist im Internet kostenlos abrufbar: http://www.gesetzeim-internet.de/urhg. tes und haben eine staatliche Erlaubnis. Gesetzliche Grundlage ist das Urheberrechtswahrnehmungsgesetz.

Für die Einrichtungen der Freien Wohlfahrtspflege sind derzeit vor allem die GEMA, VG Media, VG Wort, GVL, VG Musikedition relevant.

- In der GEMA (Gesellschaft für musikalische Aufführungs- und mechanische Vervielfältigungsrechte) sind Komponisten, Textdichter, Musiker und Musikverleger zusammengeschlossen, um ihre Verwertungsrechte geltend zu machen. Es geht um die Nutzungsrechte an urheberrechtlich geschützter Musik, die öffentlich wiedergegeben wird.

- Die VG Media (Gesellschaft zur Verwertung der Urheber- und Leistungsschutzrechte von Medienunternehmen $\mathrm{mbH}$ ) nimmt die Urheber- und Leistungsschutzrechte von mehr als 100 privaten Fernseh - und Rundfunksendeunternehmen wahr, beispielsweise die Sender Sat.1 und Klassik Radio. Dazu gehört auch das Recht zur öffentlichen Wiedergabe von Sendungen und geschützten Werken durch Zuführung von Sendesignalen, die sogenannte Kabelweitersendung.

- Die VG Wort nimmt die Rechte von Wortautoren und ihrer Verlage wahr.
- Die GVL (Gesellschaft zur Verwertung von Leistungsschutzrechten) nimmt die Zweitverwertungsrechte für ausübende Künstler und Tonträgerhersteller wahr, insbesondere die Wiedergabe von Fernseh- und Rundfunksendungen über Tonträger.

- Die VG Musikedition nimmt die Nutzungsrechte an Ausgaben von Musikwerken und Leistungsschutzrechte an wissenschaftlichen Ausgaben vorwiegend auf dem Gebiet der Musik sowie Reprographierechte für Musiknoten wahr (hierzu siehe weiter unten). sind, mit Vereinigungen von Werknutzern, also beispielsweise den Spitzenverbänden der Freien Wohlfahrtspflege oder anderen Dachorganisationen gültige Gesamtverträge zu angemessenen Bedingungen abzuschließen.

In solchen Gesamtverträgen werden für die Verbandseinrichtungen bestimmte Sonderkonditionen auf die Normaltarife vereinbart. Als Minimum erreichbar und üblich ist in jedem Fall ein Rabatt von 20 Prozent. Die Bundesarbeitsgemeinschaft der Freien Wohlfahrtspflege konnte für den Bereich der Altenhilfe und vergleichbare Einrichtungen mit der GEMA und der VG Media beispielsweise einen Zusatzrabatt für gemeinnützige Einrichtungen in Höhe von 25 Prozent aushandeln. Darüber hinaus gibt es zu bestimmten Tarifen auch Sonderrabatte und Regelungen zur Abwicklung. Der Abschluss der Einzellizenzverträge mit der GEMA und VG Media unter Nutzung der Sonderkonditionen ist Sache der einzelnen Einrichtungen.

Die Bundesarbeitsgemeinschaft der Freien Wohlfahrtspflege hält Gesamtverträge mit der GEMA seit vielen Jahren für die Bereiche Altenhilfe und Müttergenesung und seit 2010 mit der VG Media für Senioreneinrichtungen und ähnliche Einrichtungen.

Im Gesamtvertrag zwischen der Bundesarbeitsgemeinschaft der Freien Wohlfahrtspflege und der GEMA sind für den Bereich der Einrichtungen der Altenhilfe die Konditionen der Vergütung für die öffentliche Wiedergabe von Musik in Gemeinschaftsräumen und Aufenthaltsräumen sowie bei Veran-

\section{"Auch für die Weitersendung von}

$$
\begin{aligned}
& \text { Radio- und Fernsehprogrammsignalen } \\
& \text { in einer Haus-Kabelanlage sind } \\
& \text { bestimmte Bedingungen zu erfüllen " }
\end{aligned}
$$

Die größte dieser Gesellschaften ist die GEMA. Sie wickelt ihre Aufgaben über sieben Bezirksdirektionen in Deutschland ab. Außerdem zieht sie die Vergütungen für die VG Wort, die GVL, die VG Musikedition und die VG Media bei den Nutzern der Urheberrechte ein.

Das Urheberrecht sieht vor, dass die Verwertungsgesellschaften verpflichtet staltungen, außerdem für die Weitersendung von Musik über Verteileranlagen in Zimmer und Wohneinheiten sowie weitere Musiknutzungen geregelt. Der Gesamtvertrag kann in gemeinnützigen Einrichtungen mit vergleichbarem Charakter angewendet werden.

Im Gesamtvertrag zwischen der Bundesarbeitsgemeinschaft der Freien 
Wohlfahrtspflege mit der VG Media geht es um die Vergütung für die öffentliche Weitersendung von Radio- und Fernsehprogrammsignalen von privaten Sendern in einer Haus-Kabelanlage zu einzelnen Zimmern und - soweit in den Zimmern zudem Empfangsgeräte von der Einrichtung zur Verfügung gestellt werden - für die öffentliche Wiedergabe von Sendungen und geschützten Werken durch die Zuführung der Sendesignale an die Geräte.

Die Gesamtverträge einschließlich weitergehender Hintergrundinformationen finden die Mitgliedseinrichtungen über die internen Internetseiten im Fachinformationsangebot der Wohlfahrtsverbände. Weitere Informationen sind über die Webseiten der GEMA (www. gema.de) und von VG Media (www. vgmedia.de) zu erreichen. In Kürze erscheint außerdem eine Broschüre der Bundesarbeitsgemeinschaft der Freien Wohlfahrtspflege, die auf häufig gestellte Fragen eingeht und einige Übersichten zu den Nutzungsbereichen enthält. Diese ist über die internen Seiten der Bundesarbeitsgemeinschaft der Freien Wohlfahrtspflege und der jeweiligen Spitzenverbände zu beziehen.

\section{Wenn Kinder singen wollen ... VG Musikedition}

Diese Verwertungsgesellschaft kümmert sich um die Rechte der Urheber an $\mathrm{Mu}$ siknoten. Hier sieht das Gesetz keine Befreiung vor. Das Kopieren von Musiknoten ist generell vergütungspflichtig. Die GEMA und die VG Musikedition sind in den vergangen 16 Monaten massiv auf Kindertageseinrichtungen zugegangen, um ihre Rechte geltend zu machen.

Einige Gesamtverträge einzelner Wohlfahrtsverbände und im konfessionellen Bereich vermitteln einen zwanzigprozentigen Rabatt. Aktuell verhandeln GEMA und VG Musikedition mit einigen Bundesländern um die Übernahme der Vergütungen durch die Länder. Im Bundesland Bayern ist dies bereits gelungen. Dort trägt das Land Bayern die urheberrechtlichen Kosten für das Kopieren von Noten komplett.

\section{Nicht-gewerbliche öffentliche Vorführung von Filmen}

Auch bei der nicht gewerblichen öffentlichen Vorführung von Filmen gab es Un- sicherheiten, nachdem ein kommerzieller Anbieter aus dem Bereich des Filmverleihs weitflächig Senioreneinrichtungen aufgefordert hatte, sogenannte Schirmlizenzen für die nicht-gewerbliche öffentliche Filmvorführung in Senioreneinrichtungen abzuschließen.

Für diesen Bereich gibt es bisher keine staatlich anerkannte Verwertungsgesellschaft. Die öffentliche Vorführung von Filmen (DVDs, Videos u. a.) im nicht-privaten Bereich ist grundsätzlich erlaubnis- und vergütungspflichtig. Auch hierzu gibt es eine Information der Bundesarbeitsgemeinschaft der Freien Wohlfahrtspflege, die bei den internen Fachinformationen der Webseiten der Wohlfahrtsverbände $\mathrm{zu}$ erhalten ist. In einem Informationsschreiben vom 22. November 2011 heißt es zu der Problematik:

»Leider ist der Rechteerwerb für das Vorführen von Filmen nicht einfach. Das liegt daran, dass die Rechte nicht bei einer einzigen Gesellschaft erworben werden können, sondern unterschiedliche Anbieter Filmrechte einräumen. Dabei haben nicht alle Anbieter auch alle Filmrechte im Angebot. Es ist daher bei jedem einzelnen Film zu recherchieren, bei wem die Rechte für den jeweiligen Film liegen und zu erwerben sind. Erste Ansprechpartner können die regionalen konfessionellen Medienzentralen oder kommunalen Medienzentren sein. Diese bieten außer der Möglichkeit, den Film für eine Vorführung zu entleihen, oftmals auch die Möglichkeit, den Film zusammen mit dem Vorführungsrecht zu kaufen, wenn ein Film öfter gezeigt werden soll. Die Gebühren sind regelmäßig deutlich geringer, unter Umständen sogar kostenfrei. Zusätzlich wird oftmals Hintergrundmaterial zur didaktischen Arbeit angeboten. Auf Nachfrage können nichtkonfessionell gebundene Einrichtungen auch Kunde bei konfessionellen Anbietern werden. Eine Auflistung von Landes-, Stadtund Kreisbildstellen sowie der Medienzentren der Bundesländer ist abrufbar unter: www.wbf-mediende/m2/servlce/ verleihadressenhtml. Viele Anbieter sind gut miteinander vernetzt und verweisen gerne an den zuständigen Rechteinhaber weiter, wenn sie den Film selbst nicht im Angebot haben.«

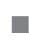

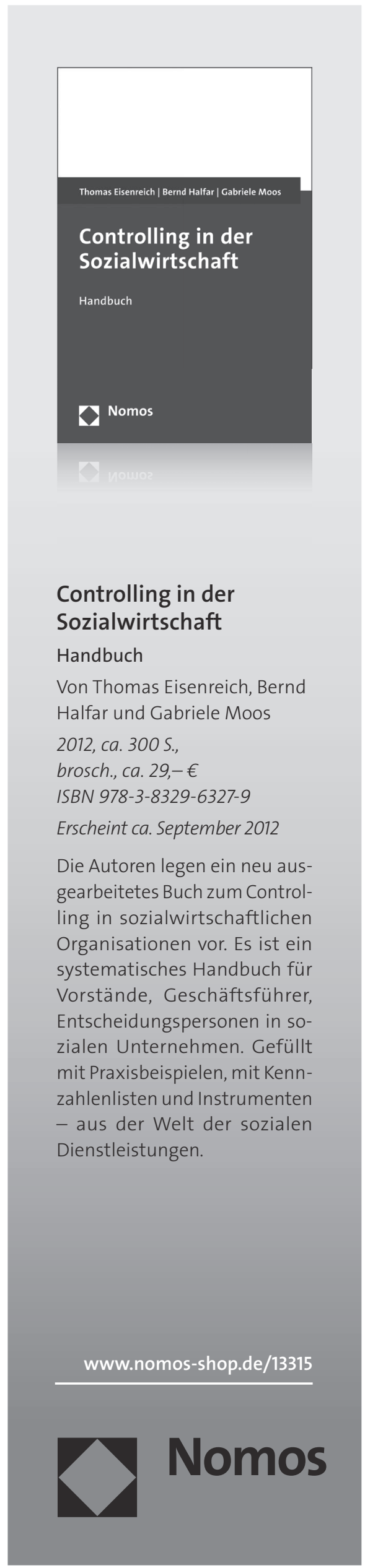

